\title{
Nicotine dependence and illness severity in schizophrenia
}

\author{
Rajeev Krishnadas, Sameer Jauhar, Susan Telfer, Somashekara Shivashankar \\ and Robin G. McCreadie
}

\section{Background}

Reasons for the increased prevalence of cigarette smoking in schizophrenia are unclear. Studies assessing clinical symptoms have sampled heterogeneous populations, with discrepant findings.

\section{Aims \\ To examine the relationship between clinical features, social adjustment and nicotine dependence in a geographically defined population of people with schizophrenia. \\ Method \\ Cross-sectional clinical study of 131 people with schizophrenia in Nithsdale, Scotland}

\section{Results}

Smokers were younger, mostly males and three times more likely to be unemployed. Those with severe nicotine dependence had greater scores on the positive subscale of the Positive and Negative Syndrome Scale (PANSS), and were prescribed higher doses of antipsychotic. Those with mildmoderate dependence had greater scores on the PANSS negative subscale. Greater symptom severity was associated with poorer social adjustment. Psychopathology and social adjustment were similar in quitters and never-smokers.

\section{Conclusions}

Our findings indicate an association between nicotine dependence, clinical symptoms and social adjustment in schizophrenia. Although causal links cannot be inferred, identifying the relationship between nicotine dependence and psychopathology may have some value in the management of smoking in schizophrenia. Further longitudinal studies are required to explore this relationship.

\section{Declaration of interest}

None.
Smoking is common in patients with schizophrenia, with one meta-analysis estimating prevalence at $62 \%$, nearly three times higher than in the general population. ${ }^{1}$ Smoking is one of the contributory factors to the starkly increased standardised mortality ratio in schizophrenia (approximately double that of the general population). Even within this population, smoking doubles the risk of cardiac-related death. ${ }^{2}$ Long-term smoking cessation rates are relatively low, and people with schizophrenia have more difficulty in quitting smoking compared with the general population. ${ }^{2}$

Prevalence studies examining the association between symptom domains and smoking have found conflicting results, with some studies suggesting increased positive and negative symptoms, ${ }^{3}$ some suggesting no difference in symptomatology between smokers and non-smokers, ${ }^{4,5}$ and a few suggesting links to either positive or negative symptoms. ${ }^{6,7}$ The small minority of studies that have measured 'nicotine dependence ${ }^{3,6-8}$ have included mainly in-patients or consecutive out-patients receiving routine care. As far as we are aware, no study has attempted to study the association between clinical variables in patients with schizophrenia and nicotine dependence in an epidemiologically defined geographical area. We therefore conducted a crosssectional survey of clinical measures and measures of nicotine dependence in Nithsdale, Scotland.

\section{Method}

\section{Sample}

The study was approved by the Dumfries and Galloway research ethics committee. On 1 February 2006, all those with a clinical diagnosis of schizophrenia according to ICD- $10^{9}$, and with a home address in Nithsdale, Scotland, were identified. Diagnosis was ascertained on the basis of the 'key informant' method, which involved identifying all patients with a diagnosis of schizophrenia known to Crichton Royal Hospital. This method has been described in detail elsewhere. ${ }^{10}$

\section{Measures}

Participants underwent a clinical interview by two of the authors (S.S. and S.T.), within 15 months of the patient census of 1 February 2006. Symptoms of schizophrenia were assessed using the Positive and Negative Syndrome Scale (PANSS). ${ }^{11}$ Total scores on individual items and each subscale were used as continuous measures in determining severity of symptoms. All participants who smoked were administered the Fagerström Test for Nicotine Dependence (FTND), and classified with high/severe dependence (FTND $>6$ ) or mild-moderate dependence (FTND $<6$ ), as defined in previous studies. ${ }^{6,12,13}$

Participants were assessed for the presence of tardive dyskinesia using the Abnormal Involuntary Movement Scale (AIMS), ${ }^{14}$ and for akathisia and extrapyramidal symptoms using the Barnes Akathisia Scale ${ }^{15}$ and Simpson-Angus Scale (SAS) ${ }^{16}$ respectively. Individuals were also classified into those with and without the deficit syndrome of schizophrenia, using the Proxy for the Deficit Syndrome criteria. ${ }^{17}$

Social adjustment in the community was assessed using the Social Adjustment Scale-Self Report (SAS-SR). ${ }^{18}$ It has 52 items, covering 'patient's role performance, interpersonal relationships, friction, feelings and satisfaction in work, and social and leisure activities with the extended family, as a spouse, parent, and member of a family unit. ${ }^{18}$ The questionnaire was completed with the supervision of a psychiatrist (S.S. or S.T.). Greater scores suggest poorer social adjustment. We used the overall SAS-SR score to see whether there was an association between social adjustment, PANSS and FTND scores. Prescriptions were converted to defined daily dose (DDD) units in order to calculate quantity of use across 
formulations. The DDD system is promoted by the World Health Organization, ${ }^{19}$ allowing the quantity of medications received to be expressed as number of days' supply of medication received at a standardised maintenance dose. Participants also completed a semi-structured questionnaire, asking for details of their smoking use, whether smoking helped them in any way, and whether they had intentions of quitting smoking. This study was part of a larger project, and the raters were unaware of the hypothesis during the study period.

\section{Statistical analysis}

Analysis was performed using SPSS version 17 for Windows. Differences between groups on categorical variables were tested using either the chi-squared test or Fisher's exact test. According to a priori hypotheses, we planned to examine the relationship between smoking and psychopathology (PANSS) using multivariate general linear model approaches. We covaried for age, gender, SAS-SR, DDD and type of antipsychotic, with family-wise error multiple testing corrections where appropriate.

Mediation is the process where the observed association between an 'explanatory' variable and 'outcome' variable occurs via a third 'mediator' variable. In our analysis, we found that greater nicotine dependence was associated with greater positive symptoms. Greater positive symptoms were also associated with poorer social adjustment (SAS-SR scores). We therefore wished to examine whether the relationship between nicotine dependence and SAS-SR scores was explained indirectly by greater positive symptoms in patients with severe nicotine dependence. In order to examine this indirect effect, we conducted a mediation analysis. The mediation analysis tests the hypothesis that a proportion of variance in the dependent variable (social adjustment) that is predicted by variance in an independent variable (nicotine dependence) can be accounted for by the mediator variable (positive symptoms). In other words, 'the mediation analysis partitions the variance explained by the predictor into a part that is independent of the mediating variable, and a part that is accounted for via the mediating variable. ${ }^{20}$

We conducted a bootstrap analysis, using Preacher and Hayes' indirect macro for SPSS, to examine the indirect effect of nicotine dependence (independent variable) and SAS-SR scores (dependent variable) through positive symptoms scores (mediator). For each analysis, 10000 random samples of the original size are taken from the obtained data, replacing each value as it was sampled, and the indirect effect $\left(a^{*} b\right)$ is computed in each sample (bootstrapping). The point estimate (mean $a^{\star} b$ ) of the indirect effect is computed over the samples. Confidence intervals are then derived from the standard errors obtained (with $Z$-score based and accelerated corrections for bias due to the underlying distribution). This analysis requires no assumption regarding the underlying distributions, since the statistical significance level is determined non-parametrically. ${ }^{21}$

\section{Results}

\section{Demographics and clinical measures}

Demographic details, according to smoking status, are shown in Table 1. Two hundred and five people with a diagnosis of schizophrenia were identified, giving a point prevalence of 3.59/ 1000 general population. Two people $(1 \%)$ were unable to give valid consent because of cognitive decline. Although they were living in the Nithsdale area on the census day, 4 individuals (2\%) had died by the time of clinical interview, and another $6(3 \%)$ had moved to a different region. Sixty-two people (30\%) refused to take part in the study. A total of 131 individuals (64\%) were therefore interviewed, and of these, $70(53.4 \%)$ were current smokers. Of the 61 who did not smoke, 21 had a history of smoking. In smokers, using the FTND cut-off score of $>6$ to diagnose severe nicotine dependence, 50 (71\%) fulfilled criteria for severe dependence and 20 (29\%) for mild-moderate dependence.

Smokers were significantly younger than non-smokers ( $t=-2.92 ; P=0.004$ ), more likely to be male (odds ratio $(\mathrm{OR})=2.94 ; 95 \%$ CI $1.44-6.00)$ and unemployed $(\mathrm{OR}=3.31$; $95 \%$ CI 1.53-7.12). Mean duration of smoking was 34.35 years (s.d. $=15.15)$. There was no significant difference in mean DDD, chlorpromazine equivalent dose, duration of illness or presence of the deficit syndrome between smokers and non-smokers. The PANSS positive scores correlated negatively with age $(r=-0.31$; $P<0.001)$. There was no difference in PANSS scores between males and females. Significantly more non-smokers reported akathisia $(\mathrm{OR}=2.22 ; 95 \%$ CI $1.83-2.7)$, with no difference in other extrapyramidal symptoms or between the two groups.

\section{Nicotine dependence}

Defined daily dose of medication was greater in the severely dependent group compared with the mild-moderate group (mean




Table 2 Difference between the groups on PANSS scores ${ }^{a}$

\begin{tabular}{|c|c|c|c|c|c|c|c|c|}
\hline & $\begin{array}{c}\text { Non-smoker } \\
(n=61)\end{array}$ & $\begin{array}{c}\text { Mild } \\
\text { dependence } \\
(n=20)\end{array}$ & $\begin{array}{c}\text { Severe } \\
\text { dependence } \\
(n=50)\end{array}$ & $F$ & $P$ & $\begin{array}{c}\mathrm{N}-\mathrm{M} \\
\text { Mean } \\
\text { difference }(P)\end{array}$ & $\begin{array}{c}\mathrm{N}-\mathrm{S} \\
\text { Mean } \\
\text { difference }(P)\end{array}$ & $\begin{array}{c}\mathrm{M}-\mathrm{S} \\
\text { Mean } \\
\text { difference }(P)\end{array}$ \\
\hline Age, years: mean (s.d.) & $57.79(17.20)$ & $54.65(17.86)$ & $47.60(12.54)$ & 4.73 & 0.01 & $2.44(0.90)$ & $9.17(0.01)$ & $6.7(0.29)$ \\
\hline $\begin{array}{l}\text { Defined daily dose of antipsychotic, } \\
\text { mean (s.d.) }\end{array}$ & $1.29(0.86)$ & $1.017(0.76)$ & $1.71(1.11)$ & 4.72 & 0.01 & $0.27(0.60)$ & $-0.42(0.06)$ & $-0.7(0.02)$ \\
\hline $\begin{array}{l}\text { Duration of illness, years: } \\
\text { mean (s.d.) }\end{array}$ & $26.00(16.70)$ & $22.00(12.69)$ & $19.68(11.42)$ & 1.96 & 0.14 & $4(0.81)$ & $6.31(0.15)$ & $2.3(0.95)$ \\
\hline Duration of smoking, years: mean (s.d.) & & $38.50(17.3)$ & $32.06(12.08)$ & $t=1.7$ & 0.10 & & & \\
\hline $\begin{array}{l}\text { People taking atypical antipsychotic, } \\
n(\%)\end{array}$ & $47(77)$ & $14(70)$ & $37(74)$ & $\chi^{2}=0.43$ & 0.81 & & & \\
\hline SAS-SR overall score & $2.10(0.56)$ & $2.3(0.52)$ & $2.13(0.51)$ & 1.10 & 0.334 & & & \\
\hline PANSS Positive score ${ }^{b}$ & $10.38(0.53)$ & $10.01(0.92)$ & $12.46(0.60)$ & 3.96 & 0.02 & 0.37 & $-2.07 *$ & -2.45 \\
\hline PANSS Negative score ${ }^{b}$ & $13.32(0.65)$ & $16.56(1.13)$ & $14.10(0.73)$ & 3.08 & 0.04 & $-3.22^{*}$ & -0.78 & 2.47 \\
\hline \multicolumn{9}{|c|}{$\begin{array}{l}\text { N, non-smoker; M, mild dependence; S, severe dependence; SAS-SR, Social Adjustment Scale - Self Report; PANSS, Positive and Negative Syndrome Scale. } \\
\text { a. Only items with a significant difference are shown. } \\
\text { b. Multivariate analysis of covariance with PANSS subscores as dependent variables; covariates appearing in the model are evaluated at the following values: SAS-SR overall }=2.14 \text {, } \\
\text { gender }=0.45 \text {, defined daily dose }=1.41 \text {, type of antipsychotic }=0.21 \text {, age }=53.22 \text {. Post-hoc tests are based on estimated marginal means and adjusted for multiple comparisons } \\
\text { using Bonferroni family-wise error correction. } \\
\text { *Significant at } P<0.05 \text {. }\end{array}$} \\
\hline
\end{tabular}

difference $-0.7 ; P=0.019)$, although the difference compared with non-smokers did not reach statistical significance (mean difference $-0.42 ; P=0.06$ ) (Table 2). There was no difference in duration of illness and antipsychotic type among the three groups.

\section{PANSS subscale scores and nicotine dependence}

Multivariate analysis of covariance was used to examine the relationship between non-smokers $(n=61)$, smokers with severe dependence ( $>6$ on FTND, $n=50$ ), mild dependence $(<6$ on FTND, $n=20)$ and PANSS scores. Nicotine dependence status significantly predicted PANSS scores (Wilk's lambda 0.875; $F(6,242)=2.78 ; P=0.01)$. On tests of between-participants effects, dependence status significantly predicted total positive scores $(F(2,123)=3.96 ; \quad P=0.02) \quad$ and total negative scores $(F(2,123)=3.08 ; \quad P=0.04)$, but not general psychopathology scores $(F(2,123)=0.092 ; P=0.9)$.

We conducted post hoc pair-wise comparisons of the total scores between non-smokers, those with mild dependence and those with severe dependence, using Bonferroni family-wise error multiple testing corrections. Those with severe dependence had greater positive scores compared with non-smokers $(t=2.72$; $P=0.001)$. Those with mild dependence had greater total negative symptoms scores compared with non-smokers $(t=3.72 ; P=0.007)$ (Table 2). This was strengthened by the finding that $35 \%$ of those with mild dependence had deficit syndrome compared with $18 \%$ of non-smokers and $26 \%$ of those with severe nicotine dependence. This difference did not reach statistical significance.

\section{Smoking severity, PANSS scores and social adjustment}

There was a significant association between SAS-SR overall scores and PANSS positive $(r=0.25 ; P=0.004)$, negative $(r=0.34$; $P<0.001)$ and general psychopathology $(r=0.47 ; \quad P<0.001)$ scores. There was no direct statistically significant association between SAS-SR scores and smoking dependency $(r=0.18$; $P=0.84)$. However, there was an indirect effect, where greater PANSS positive symptom score mediated the relationship between greater nicotine dependence and worse overall SAS-SR scores (effect $=0.01 ; Z=2.10$; s.e. $=0.0043 ; 95 \%$ CI $0.003-0.02)$.

We compared quitters and never-smokers (21 out of 61 nonsmokers had a history of nicotine use). Quitters did not differ from never-smokers in terms of positive symptoms (mean difference $0.51 ; P=0.9$ ), negative symptoms (mean difference
2.6; $P=0.11$ ) or SAS-SR scores (mean difference $0.16 ; P=0.76$ ) and DDD (mean difference $0.14 ; P=0.9$ ).

Among smokers, $42(60 \%)$ stated that it helped them to relax, $11(16 \%)$ stated that they smoked because they felt lonely, 10 (14\%) that it helped them to socialise better and 22 (31\%) stated that they smoked because they felt anxious or depressed. Fifty-six smokers (80\%) said that they enjoyed smoking. In total, $22(31 \%)$ claimed that they tried to give up smoking in the past 12 months, $21(30 \%)$ had a desire to give up and $13(19 \%)$ said they had an intention to give up.

\section{Discussion}

In an epidemiologically defined sample of patients with schizophrenia, we found that smoking rates in patients $(53.4 \%)$ were twice that seen in the general population in Dumfries and Galloway (26.4\% in men and $24.9 \%$ in women). ${ }^{22}$ Smoking rates in this population do not appear to have changed significantly since they were last measured over 20 years ago $(58 \%),{ }^{23}$ a finding echoed in a recent prospective study. ${ }^{4}$

Patients with severe nicotine dependence had greater positive symptoms and were prescribed higher doses of antipsychotic medication. Negative symptoms were greater in those with mild dependence (compared with non-smokers and those with severe dependence), a finding that was confirmed by increased rates of deficit syndrome in this group. This is in contrast to previous findings from Nithsdale, which did not find a relationship between symptomatology and smoking status. However, that study used the Manchester Scale to identify psychopathology, and did not measure nicotine dependence. ${ }^{23}$

Greater positive and negative symptoms were associated with poorer social adjustment. We also found an indirect effect of nicotine dependence on overall social adjustment, suggesting that nicotine dependence may be associated with poorer social adjustment indirectly through its association with greater positive symptoms. This is further supported by our finding that patients with severe nicotine dependence were more likely to be unemployed and receiving greater doses of antipsychotic medication. The higher dosages of medications in patients with severe dependence may reflect either increased rate of antipsychotic metabolism and/or the need for more aggressive treatment of positive symptoms. Furthermore, quitters and never-smokers did not differ on measures of psychopathology, DDD or social adjustment. 
These results, however, should be interpreted with caution. Although classic mediation analysis tests causal relationships between variables, such conclusions cannot be made owing to the cross-sectional nature of our data. Most patients in our sample started smoking before the onset of their illness. However, we do not know the temporal relationship between nicotine dependence, symptomatology and social adjustment. It is possible that greater dependence, greater medication dosage and poorer social adjustment are epiphenomena associated with greater symptomatology in this group.

Similar to previous findings from Nithsdale, those who smoked in the present cohort were more likely to be male and significantly younger. Younger people also had greater PANSS positive scores. As there is some evidence suggesting that positive symptoms become less prominent with age, it is tempting to argue that the presence of younger patients in the sample was the reason for the greater positive symptoms scores in the smoking group. ${ }^{24}$ However, the relationship between nicotine dependence and positive symptoms remained after controlling for age. Duration of illness between smokers and non-smokers did not differ in our population. This finding replicates those of a recent metaanalysis, which suggests that the age at onset of psychosis did not differ significantly between smokers and non-smokers. ${ }^{25}$

\section{Strengths and limitations}

This clinical study was conducted in a defined population of people with schizophrenia, with an attempt to minimise selection bias. Self-report of smoking status is viewed as a reliable measure, ${ }^{26}$ and efforts were made to measure not only smoking status, but also a measure of nicotine dependence (FTND), acknowledging that dependence is independent of the number of cigarettes smoked, level of nicotine within cigarettes or duration of smoking. ${ }^{27}$ Furthermore, another study has found the FTND to have a strong association with DSM-IV diagnosed nicotine dependence $(r=0.89, P<0.001) .^{7}$ Although some authors have pointed to poor psychometric properties of the FTND in patients with schizophrenia, ${ }^{28}$ the study most often quoted in this regard found an internal consistency of 0.74 in smokers with schizophrenia (higher than in smoking controls). ${ }^{29}$ Limitations of our study include its cross-sectional nature, and therefore the direction of causality cannot be ascertained. Regarding antipsychotic medication, we did not measure concordance through use of other measures (e.g. pill count, corroborative history), although measures of adherence are not routinely used in clinical studies. Another limitation is the absence of clinical measures (other than the PANSS) measuring psychiatric comorbidity in more depth (e.g. anxiety and depression) or cognitive functioning. A substantial proportion of people refused to take part in the study, another source of selection bias that is inherent in clinical studies in a 'real world' setting. Examining the differences between those who participated and those who did not, revealed that individuals living in supported accommodation were more accessible and were more willing to participate, while the relatively 'higher functioning' group who were employed and living with family, with lesser duration of illness, refused to take part in the study.

\section{Measuring nicotine dependence in patients with schizophrenia}

The majority of the clinical literature on smoking and schizophrenia has focused solely on smoking status, with conflicting results, that may reflect differences in sample selection.

To our knowledge, only four other studies have measured nicotine dependence (each utilising the FTND), although only one of these studies analysed differences in groups, based on a categorical measure of nicotine dependence, ${ }^{6}$ finding lower total PANSS and positive PANSS scores in mildly dependent smokers compared with severely dependent smokers and non-smokers. In that study, individuals with severe dependence had worse outcome, and in one of the other studies, increasing FTND scores correlated with poorer Clinical Global Impression score. ${ }^{8}$ It is worth noting that an almost consistent feature of clinical studies, including the present study, examining smoking in schizophrenia is the poorer outcome in smoking groups. ${ }^{3,6}$ Our study found that patients who smoked were three times more likely to be unemployed, and those with greater dependence were also prescribed greater doses of antipsychotic medication. The finding of increased antipsychotic dose in smokers is well recognised; for example, Salokangas et al found an increased antipsychotic dosage in older patients with schizophrenia who smoked, with higher plasma levels of unmetabolised fractions of antipsychotic detected. ${ }^{30}$ Parkinsonian symptoms did not differ among the groups in our study. However, compared with smokers, patients who did not smoke were twice as likely to develop akathisia. Lower scores on the Barnes Akathisia Scale in smokers with schizophrenia have been reported elsewhere, ${ }^{5}$ and were also found in a placebo-controlled trial of nicotine transdermal patches applied to patients with schizophrenia treated with haloperidol. ${ }^{31}$ Our findings are similar to those of Zhang et al who found no association between tardive dyskinesia and smoking status. ${ }^{32}$

\section{Examining the relationship between nicotine dependence and schizophrenia}

Various theories have been put forward for the association between smoking and schizophrenia. ${ }^{33}$ The two that are most often cited are the self-medication and shared vulnerability hypotheses.

\section{Nicotine as self-medication}

Originally proposed over 25 years ago as a way of understanding substance dependence, the self-medication hypothesis has been increasingly used to explain the high association between substance misuse and severe mental illness. With regard to smoking, the mechanisms include possible regulation of central dopamine availability by pharmacodynamic and pharmacokinetic processes (e.g. enzyme induction of the hepatic enzyme system, lowering antipsychotic effectiveness). These processes have also been implicated in reducing the side-effects of dopamine blockade of typical antipsychotics. More recently, based on a number of preclinical and clinical studies, this theory proposes that patients with schizophrenia smoke in order to 'treat' underlying negative symptoms and cognitive deficits. ${ }^{34,35}$

Nicotinic receptors are present on ventral tegmental dopaminergic cell bodies, with nicotine inducing dopamine release in the ventral tegmental system. Nicotine-induced release of dopamine in the mesocortical system results in improved cognitive performance and a decrease in negative symptoms. ${ }^{36}$ The effects of smoking on the mesolimbic system are more complex. Evidence from human positron emission tomography studies suggests it increases the release of dopamine in the dorsal/ventral striatum (to a similar degree as other drugs of misuse). ${ }^{37}$ This, along with increased metabolism of antipsychotics may be a plausible explanation for our finding of increased positive symptoms in our population, given the dopamine hypothesis that links positive psychotic symptoms to increased release of dopamine in the limbic system. ${ }^{38}$ Greater negative symptoms in those with mild dependence compared with severe dependence, could suggest a trajectory where those with 
severe dependence have successfully overcome negative symptoms by increasing their level of nicotine dependence. This seems to come at a price: increased positive symptoms, treatment with greater antipsychotic dose and poorer social adjustment. Whether a dose-response relationship exists between nicotine and psychopathology, cognitive symptoms or side-effects of medication is not clear. In our study, a significant majority of patients said they enjoyed smoking, that it helped them relax and socialise better. This suggests that a significant proportion of people perceive some benefit from smoking.

A number of neurophysiological studies have provided evidence for the self-medication hypothesis. Normalisation of the auditory sensory gating (P50) abnormalities, prepulse inhibition abnormalities and eye-tracking deficits following nicotine administration in patients with schizophrenia, and in relatives of patients, have all pointed towards a biological basis underlying the self-medication hypothesis of schizophrenia. ${ }^{33}$ Similarly, Jacobsen et al using functional magnetic resonance imaging showed that nicotine enhances functional connectivity between regions of the brain that mediate a working memory task. ${ }^{39}$ However, the relationship between smoking, negative and cognitive symptoms is complex. In a recent case-control study, Zhang et al found that although smokers had lower negative symptoms compared with non-smokers, they performed poorly on cognitive functioning, as measured by the Repeatable Battery for the Assessment of Neuropsychological Status (RBANS). ${ }^{40}$ It should be noted that RBANS is not the most robust measure of frontal/executive function. ${ }^{41}$

\section{Nicotine as shared vulnerability}

In our study, smoking pre-dated the onset of illness. However, the relationship between nicotine dependence and duration of prodromal or subsyndromal symptoms preceding the onset of illness are not known. This finding suggests that self-medication may not be the only possible reason for the increased rates of nicotine dependence in this population. Previous studies have put forward a shared vulnerability hypothesis between nicotine and schizophrenia.

In this context, Esterberg et al found that first-degree relatives of patients with schizophrenia, with greater schizotypy, were more likely to smoke. ${ }^{42}$ There was no relationship between levels of schizotypy and smoking status in the control group, despite having similar levels of schizotypy. This finding is relevant, as the only difference between relatives and controls was the genetic vulnerability in the relatives. Esterberg et al therefore proposed that having a genetic vulnerability to schizophrenia may also place an individual at risk for smoking cigarettes. Furthermore, a number of studies have shown a shared genetic vulnerability to both schizophrenia and nicotine dependence. ${ }^{43}$ Genetic and human post-mortem studies have shown that both high- and low-affinity nicotinic receptor expression is reduced in several brain regions in people with schizophrenia, even in those with a history of smoking (an effect opposite to that seen in normal smokers). The 15q14 locus near the coding region of the CHRNA7 gene (coding for the neuronal acetylcholine receptor $\alpha 7$ subunit, $\alpha 7-n A C h R$ protein) has been consistently linked to schizophrenia and sensory gating abnormalities, especially the deficient P50 suppression (see Wing et al for a recent review of the literature). ${ }^{44}$ A recent preclinical study found that reduction in $\alpha 7-\mathrm{nAChR}$ is associated with the motivation for nicotine self-administration. Together, these findings suggest that reduction in $\alpha 7-n A C h R$ function (a core deficit in patients with schizophrenia), promotes nicotine use in patients with schizophrenia. ${ }^{45}$

Whether nicotine use is a result of self-medication, or a shared vulnerability, outcomes appear worse in this group of patients. Our findings also suggest that reasons for smoking (and the development of dependence) in schizophrenia are possibly multifactorial (Fig. 1.).

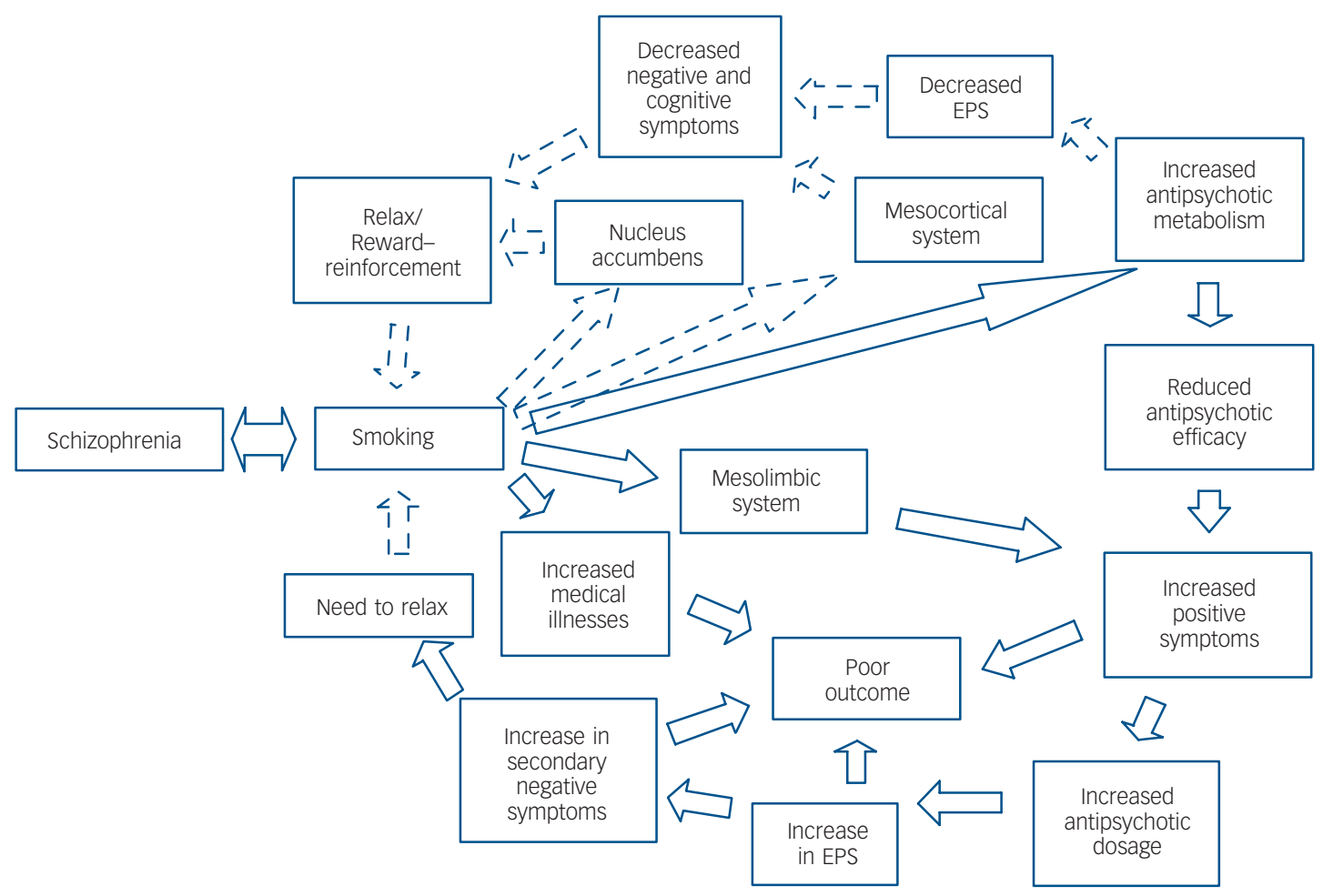

Fig. 1 Reasons for smoking and the development of dependence in schizophrenia.

Dashed arrows depict the self-medication hypothesis whereby smoking improves negative and cognitive symptoms and side-effects. The continuous arrows represent pathways that may lead to poor outcomes. Double-sided arrow suggests a shared vulnerability. EPS, extrapyramidal symptoms. 


\section{Clinical implications}

Rates of smoking in patients with schizophrenia were greater than in the general population and do not appear to have changed significantly over the past 20 years. A rather disconcerting finding in our study was that less than a quarter of the sample had an intention of giving up smoking. On a positive note, about $25 \%$ of those with a lifetime history of smoking had quit, and clinically they did not differ from lifetime non-smokers.

Nicotine dependence was found to be associated with symptom severity and outcome in people with schizophrenia. Although our study does not establish a causal relationship between these variables, identifying and treating nicotine dependence may have some value in reducing morbidity and mortality in schizophrenia. People with schizophrenia who smoke have been shown to benefit from pharmacological and psychosocial interventions that target smoking. When patients are assessed in view of quitting smoking, care should be taken to delineate the relationship between nicotine dependence and symptom severity, as this may potentially interfere with a person's successful attempt at quitting smoking. Further systematic longitudinal studies are required to establish whether a causal link exists between nicotine dependence and psychopathology.

\section{Acknowledgements}

We would like to thank all study participants.

Rajeev Krishnadas, MBBS, MD, DipNB, MRCPsych, Sackler Institute of Psychobiological Research, University of Glasgow, Southern General Hospital, Glasgow: Sameer Jauhar, MBChB, BSC (Hons), MRCPsych, Department of Psychosis Studies, Institute of Psychiatry, King's College London; Susan Telfer, MBBS, MRCPsych, Coathill Hospital, NHS Lanarkshire; Somashekara Shivashankar, MBBS, MRCPsych, Cardiff and Vale University Local Health Board, Psychiatry, Cardiff; Robin G. McCreadie, DSC, MD, FRCPsych, Crichton Royal Hospital, Psychiatry, Dumfries, UK

Correspondence: Rajeev Krishnadas, Sackler Institute of Psychobiological Research, University of Glasgow, Southern General Hospital, Glasgow G51 4TF, UK. Email: Rajeev.Krishnadas@glasgow.ac.uk

First received 20 Dec 2011, final revision 9 Jun 2012, accepted 27 Jun 2012

\section{References}

1 deLeon J, Diaz F. A meta-analysis of worldwide studies demonstrates an association between schizophrenia and tobacco smoking behaviors. Schizophr Res 2005; 76: 135-57.

2 Kelly DL, McMahon RP, Wehring HJ, Liu F, Mackowick KM, Boggs DL, et al. Cigarette smoking and mortality risk in people with schizophrenia. Schizophr Bull 2011; 37: 832-8

3 Kao Y-C, Liu Y-P, Cheng T-H, Chou M-K. Cigarette smoking in outpatients with chronic schizophrenia in Taiwan: relationships to socio-demographic and clinical characteristics. Psychiatr Res 2011; 190: 193-9.

4 Kotov R, Guey LT, Bromet EJ, Schwartz JE. Smoking in schizophrenia: diagnostic specificity, symptom correlates, and illness severity. Schizoph Bull 2008; 36: 173-81.

5 Barnes M, Lawford B, Burton S, Heslop K, Noble E, Hausdorf K, et al. Smoking and schizophrenia: is symptom profile related to smoking and which antipsychotic medication is of benefit in reducing cigarette use? Aust $N \mathrm{ZJ}$ Psychiatry 2006; 6-7: 575-80.

6 Aguilar MC, Gurpegui M, Diaz FJ, De Leon J. Nicotine dependence and symptoms in schizophrenia: Naturalistic study of complex interactions. Br J Psychiatry 2005; 186: 215-21.

7 Patkar A, Gopalakrishnan R, Lundy A, Leone F, Certa K, Weinstein S. Relationship between tobacco smoking and positive and negative symptoms in schizophrenia. J Nerv Ment Dis 2005; 190: 604-10.

8 Herran A, deSantiago A, Sandoya M, Fernandez M, Dez-Manrique J, Vazquez-Barquero J. Determinants of smoking behaviour in outpatients with schizophrenia. Schizophr Res 2000; 41: 373-81.
9 World Health Organization. The ICD-10 Classification of Mental and Behavioural Disorders: Clinical Descriptions and Diagnostic Guidelines. WHO, 1992.

10 Telfer S, Shivashankar S, Krishnadas R, McCreadie RG, Kirkpatrick B. Tardive dyskinesia and deficit schizophrenia. Acta Psychiatr Scand 2011; 124: 357-62.

11 Kay SR, Fiszbein A, Opler LA. The positive and negative syndrome scale (PANSS) for schizophrenia. Schizophr Bull 1987; 13: 261-76.

12 de Leon J, Diaz FJ, Becona E, Gurpegui M, Jurado D, Gonzalez-Pinto A. Exploring brief measures of nicotine dependence for epidemiological surveys. Addict Behav 2003; 28: 1481-6.

13 Fagerstrom KO, Kunze M, Schoberberger R, Breslau N, Hughes JR, Hurt RD, et al. Nicotine dependence versus smoking prevalence: comparisons among countries and categories of smokers. Tob Control 1996; 5: 52-6.

14 Lane RD, Glazer WM, Hansen TE, Berman WH, Kramer SI. Assessment of tardive dyskinesia using the Abnormal Involuntary Movement Scale. J Nerv Ment Dis 1985; 173: 353-7.

15 Barnes TR. A rating scale for drug-induced akathisia. Br J Psychiatry 1989; 154: 672-6.

16 Simpson G, Angus J. A rating scale for extrapyramidal side effects. Acta Psychiatr Scand 1970; 212: 11-19.

17 Kirkpatrick B, Buchanan RW, Breier A, Carpenter Jr WT. Case identification and stability of the deficit syndrome of schizophrenia. Psychiatry Res 1993; 47: 47-56.

18 Weissman MM, Bothwell S. Assessment of social adjustment by patient self-report. Arch Gen Psychiatry 1976; 33: 1111-5.

19 World Health Organization. Guidelines for ATC Classification and DDD Assignment (13th edn). WHO Collaborating Centre for Drugs Statistics Methodology, 2009.

20 Palaniyappan L, Liddle PF. Differential effects of surface area, gyrification and cortical thickness on voxel based morphometric deficits in schizophrenia. Neuroimage 2012; 60: 693-9.

21 Preacher KJ, Hayes AF. Asymptotic and resampling strategies for assessing and comparing indirect effects in multiple mediator models. Behav Res Methods 2008; 40: 879-91.

22 Whyte B, Gordon D, Haw S, Fischerbacher C, Scott N, Harrison R. An Atlas of Tobacco Smoking in Scotland: A Report Presenting Estimated Smoking Prevalence and Smoking Attributable Deaths within Scotland. NHS Health Scotland, 2007.

23 Kelly C, Mccreadie R. Smoking habits, current symptoms, and premorbid characteristics of schizophrenic patients in Nithsdale, Scotland. Am J Psychiatry 1999; 156: 1751-7.

24 Tandon R, Nasrallah HA, Keshavan MS. Schizophrenia, 'just the facts' 4. Clinical features and conceptualization. Schizophr Res 2009; 110: 1-23.

25 Myles N, Newall H, Compton MT, Curtis J, Nielssen O, Large M. The age at onset of psychosis and tobacco use: a systematic meta-analysis. SOC Psychiatry Psychiatr Epidemiol 2011; Sep 8. Epub ahead of print.

26 Pierce JP, Dwyer T, DiGiusto E, Carpenter T, Hannam C, Amin A, et al. Cotinine validation of self-reported smoking in commercially run community surveys. J Chronic Dis 1987; 40: 689-95.

27 Panday S, Reddy SP, Ruiter RA, Bergstrom E, de Vries H. Nicotine dependence and withdrawal symptoms among occasional smokers. $J$ Adolesc Health 2007; 40: 144-50.

28 Levander S, Eberhard J, Lindstrom E. Nicotine use and its correlates in patients with psychosis. Acta Psychiatr Scand 2007; 116(S435): 27-32.

29 Weinberger AH, Reutenauer EL, Allen TM, Termine A, Vessicchio JC, Sacco KA, et al. Reliability of the Fagerstrom Test for Nicotine Dependence, Minnesota Nicotine Withdrawal Scale, and Tiffany Questionnaire for Smoking Urges in smokers with and without schizophrenia. Drug Alcohol Depend 2007; 86: 278-82.

30 Salokangas R, Saarijarvi S, Taiminen T, Lehto H, Niemi H, Ahola V, et al. Effect of smoking on neuroleptics in schizophrenia. Schizophr Res 1997; 23: 55-60.

31 Yang YK, Nelson L, Kamaraju L, Wilson W, McEvoy JP. Nicotine decreases bradykinesia-rigidity in haloperidol-treated patients with schizophrenia. Neuropsychopharmacology 2002; 27: 684-6.

32 Zhang $X Y$, Yu YQ, Sun S, Zhang $X$, Li W, Xiu MH, et al. Smoking and tardive dyskinesia in male patients with chronic schizophrenia. Prog Neuropsychopharmacol Biol Psychiatry 2011; 35: 1765-9.

33 Winterer G. Why do patients with schizophrenia smoke? Curr Opin Psychiatry 2010; 23: 112-9.

34 Matthews A, Wilson V, Mitchell S. The role of antipsychotics in smoking and smoking cessation. CNS Drugs 2011; 25: 299-315.

35 Kumari V, Postma P. Nicotine use in schizophrenia: The self medication hypotheses. Neurosci Biobehav Rev 2005; 29: 1021-34. 
36 George Y. Neurobiological links between nicotine addiction and schizophrenia. J Dual Diagn 2007; 3: 27-42.

37 Brody A, Olmstead R, London E, Farahi J, Meyer J, Grossman P, et al. Smoking induced ventral striatum dopamine release. Am J Psychiatry 2004 161: 1211-8.

38 Howes OD, Kapur S. The dopamine hypothesis of schizophrenia: version III - the final common pathway. Schizophr Bull 2009; 35: 549-62.

39 Jacobsen LK, D'Souza DC, Mencl WE, Pugh KR, Skudlarski P, Krystal JH. Nicotine effects on brain function and functional connectivity in schizophrenia. Biol Psychiatry 2004; 55: 850-8

40 Zhang XY, Chen da C, Xiu MH, Haile CN, Sun H, Lu L, et al. Cigarette smoking and cognitive function in chinese male schizophrenia: a case-control study. PLOS ONE 2012; 7: e36563.
41 Strauss E, Sherman EMS, Spreen O. A Compendium of Neuropsychological Tests: Administration, Norms, and Commentary (3rd edn). Oxford University Press, 2006.

42 Esterberg ML, Jones EM, Compton MT, Walker EF. Nicotine consumption and schizotypy in first-degree relatives of individuals with schizophrenia and non-psychiatric controls. Schizophr Res 2007; 97: 6-13.

43 Yoshimasu K, Kiyohara C. Genetic influences on smoking behavior and nicotine dependence: a review. J Epidemiol 2003; 13: 183-92.

44 Wing VC, Wass CE, Soh DW, George TP. A review of neurobiological vulnerability factors and treatment implications for comorbid tobacco dependence in schizophrenia. Ann N Y Acad Sci 2011; 1248: 89-106.

45 Brunzell DH, McIntosh JM. Alpha7 nicotinic acetylcholine receptors modulate motivation to self-administer nicotine: implications for smoking and schizophrenia. Neuropsychopharmacology 2011; 37: 1134-43.

\section{extra Usage of the Persian word del in psychiatry}

\section{Cyrus Abbasian}

Witness del, what love's sadness did again Became del-taker, calamity brought to faithful friend

Hâfez (14th century)

Psychosomatic symptoms are a common presentation of mental health problems in Iran. The word del, which usually means the heart and can signify love, also symbolises the stomach or abdominal region. It can be used with a plethora of words and an online dictionary (http://farsilookup.com) highlights almost 400 words incorporating del.

Del is frequently used to express anxiety and mood-related symptoms: for example, terms such as del-frenzy or del-excitement describe anxiety whereas del-intransigence is worry. Sadness is del-tightness and melancholy del-congestion. Uncertainty is two-del and worry-free is peaceful-del. Whereas cold-del means hopeless, hopeful is warm-del. Black-del is vengeful, lion-del courageous and turbulent-del upset. Whereas stone-del is cruel thin-del is sensitive.

Given the links between mental illness and bowel symptoms this connection is unsurprising. Terms such as 'stomach aches' or 'butterflies' are commonly used in English and symptoms such as constipation, diarrhoea, nausea and vomiting are related to mood and anxiety disorders. Conversely, patients referred for bowel disorders significantly suffer from higher psychosocial distress. Del-pain remains a common method of depicting psychosocial distress among Iranians. Not surprisingly, the word del also crops up frequently in Persian poetry.

The poem is the author's own translation from the original Persian. 